\title{
26. COMMISSION DES ETOILES DOUBLES
}

Président d'honneur: M. Aitken.

Président: M. W. H. van DEN Bos, Union Astronomer, Union Observatory, Johannesburg, South Africa.

Membres: M. Baize, Mme Bonnet-Sainturier, MM. B. H. Dawson, Finsen, Hertzsprung, Jeffers, Kuiper, Luyten, P. Muller, Rossiter, Russell, Strand, van Biesbroeck, Voûte, Wallenquist, Woolley, Zagar.

The effect which the war has had on the progress of Astronomy in general, and of Double Star Astronomy in particular, cannot yet be reliably estimated, but we can already say that it is severe and will be felt for many years to come. Its least serious (because, we may hope, only temporary) aspect is the delay in the publication of observations. This effect may be mitigated if observers will communicate their unpublished results and forward reprints of their publications to the two central offices: Lick Observatory for declinations $+90^{\circ}$ to $-30^{\circ}$, Union Observatory for $-19^{\circ}$ to $-90^{\circ}$. If this be done, information supplied by these offices could include unpublished results.

The completion of the systematic surveys at Lick, Bloemfontein and Johannesburg seems to have made the discovery of new pairs amongst the stars down to the ninth Durchmusterung magnitude largely a matter of the past, unless a considerable improvement in our powers of detection should be achieved. For the fainter stars, a continuation of the visual work of Espin, Jonckheere and others with instruments of greater optical power as well as inspection of photographic plates obtained with long focus instruments would doubtless result in the addition of large numbers of faint pairs. The question is: how would this benefit the progress of our branch of Astronomy, when we are not even in a position to provide adequate, systematic measurement of the pairs already known? It would seem a wiser policy to test for duplicity (repeatedly, when desirable) a limited number of stars selected for special reasons, such as large parallax or proper motion, variable stars when near minimum light, etc. If we should desire some knowledge of the occurrence of double stars among the stars fainter than the ninth magnitude, some Selected Areas plan would seem to be indicated, in order to keep the number of new discoveries within manageable proportions.

With regard to routine measurement of known pairs, the position is not satisfactory. The small number of powerful instruments which are wholly or in part devoted to this task have to carry too great a responsibility. When one keeps a Card Catalogue up to date, one knows how much remains to be done. It is not very satisfying to find that a pair such as ADS 8739 was not measured between I902 and I924, nor does a pair such as ADS 4472 give evidence of careful planning of our observing programmes.

We still have to rely mainly on visual measurement with the micrometer in one of its various forms. While the usual micrometer, if used with care and skill under adequate atmospheric conditions, still seems to give the best results as far as position angles are concerned, modern forms of the double-image micrometer and the comparison-image micrometer appear to give better distances, with the additional advantage that they can be used for determining magnitude differences. With suitable precautions, both interferometric and photographic measurement can reach a much higher accuracy than visual, but both are of limited applicability.

Orbit computation leaves nothing to be desired. Whenever a double star has been adequately measured - and not infrequently long before this stage has been reached-one or more orbits are certain to appear.

Our observations of position need to be supplemented by accurate data on parallax, mass ratio, magnitudes, spectral types, radial velocities, etc., of great importance for theoretical investigations. More attention is now being paid to this aspect, but much remains to be done here, notably for faint objects of special interest, such as pairs with degenerate components.

W. H. vAN DEN BOS President of the Commission 
Report of meeting. Friday, August I3, I948. II.30 a.m.

President: Dr W. H. van den Bos.

SeCretary: Mr P. Muller.

The President opened the meeting, Mr P. Muller acting as Secretary.

The President remarked that, as an overlap of one degree between the domains covered by the northern and southern central offices is sufficient, it might lighten the burden of the northern office if $\mathrm{Dr}$ Jeffers considered shifting his southern limit from $-30^{\circ}$ to $-20^{\circ}$.

Dr Jeffers gave information on the present state of the northern Card Catalogue at Lick Observatory, received from Dr Aitken a few years ago. As none of those present desired to discuss further points of the Draft Report, the President proceeded to read a letter from Prof. Hagihara, that the astronomers at Tokyo intend to take up the measurement of double stars as soon as the 26 -inch refractor is back in operation.

The President invited those present to send letters of greeting to Messrs R. G. Aitken (Honorary President), H. N. Russell, G. van Biesbroeck and J. Voûte. These were circulated and signed.

The desirability of a publication of General Catalogues for the northern and southern domains, to bring the ADS and SDS up to date since 1927 , was next discussed. The general opinion was that such publications, in a not too distant future, were highly desirable.

Dr Jeffers remarked that a large number of observations made in recent years had not yet become available at Lick and the President confirmed that the same applied to the southern central office.

Dr Jeffers invited opinions on the most desirable form of such a General Catalogue. The President pointed out that this was not an urgent matter at the present stage and could be dealt with more efficiently by correspondence when the time to prepare manuscripts approached.

The President invited a discussion on the future and aims of Double Star Astronomy. He mentioned that a number of experienced astronomers had voiced their concern that few young men appear to enter this field, to replace those retiring from active work. $\mathrm{He}$ himself agreed that this was the case, but was not particularly worried, as he believed that unjustified neglect of any branch of astronomy was bound to correct itself in the long run. Dr Mitchell said that the same situation was found in parallax observation and that he ascribed it to the rigours of the observational work. The President remarked that the case is relatively more serious for double stars, as these have to be kept under observation, whereas a parallax is known, once it has been sufficiently well determined. Nevertheless, he hoped that double star observation would always be in the hands of astronomers who took a genuine interest in the subject, as the results obtained by those who perform it as a routine duty tend to be of small value.

Dr Baize wished to see the technique of measurement improved. According to his comparisons, the filar micrometer is satisfactory for position angles, but surpassed by modern forms of the double image micrometer in the measurement of distances.

Prof. Zagar mentioned the great need for accurate determination of differences of magnitude and radial velocities, as referred to in the Draft Report, and emphasized the importance of continued statistical studies of double stars.

Mr Muller stated that, using his double image micrometer as a photometer, he is obtaining magnitude differences with a mean error of about $0^{\mathrm{m}} \cdot 04$.

Prof. R. E. Wilson said that astronomers working in other branches needed the results of double star observers and that at Mount Wilson spectra of both components of about 200 pairs have been obtained. This permits them to be placed on a Russell diagram. Mr Muller mentioned that a similar result was obtained from his measurements of magnitude differences in two colours, red and green, for a number of bright stars.

There being no further discussion, the President closed the meeting at I2.I5 p.m. 